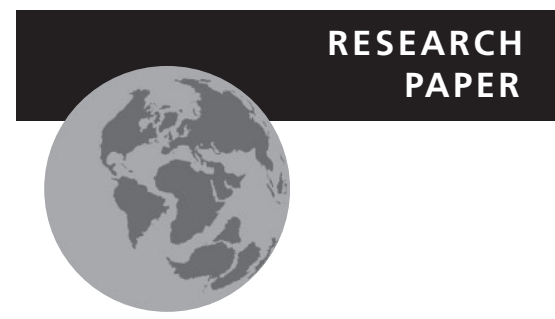

\title{
Predicting richness and composition in mountain insect communities at high resolution: a new test of the SESAM framework
}

Manuela D'Amen ${ }^{1 \star}$, Jean-Nicolas Pradervand ${ }^{1}$ and Antoine Guisan ${ }^{1,2}$

${ }^{1}$ Department of Ecology and Evolution, University of Lausanne, Biophore Building, 1015 Lausanne, Switzerland, ${ }^{2}$ Institute of Earth Surface Dynamics, Geopolis, University of Lausanne, 1015 Lausanne, Switzerland
${ }^{*}$ Correspondence: Manuela D’Amen, Department of Ecology and Evolution, University of Lausanne, Biophore Building, 1015 Lausanne, Switzerland.

E-mail: manuela.damen@unil.ch

\section{ABSTRACT}

Aim The aim of this study was to test different modelling approaches, including a new framework, for predicting the spatial distribution of richness and composition of two insect groups.

Location The western Swiss Alps.

Methods We compared two community modelling approaches: the classical method of stacking binary prediction obtained from individual species distribution models (binary stacked species distribution models, bS-SDMs), and various implementations of a recent framework (spatially explicit species assemblage modelling, SESAM) based on four steps that integrate the different drivers of the assembly process in a unique modelling procedure. We used: (1) five methods to create bS-SDM predictions; (2) two approaches for predicting species richness, by summing individual SDM probabilities or by modelling the number of species (i.e. richness) directly; and (3) five different biotic rules based either on ranking probabilities from SDMs or on community co-occurrence patterns. Combining these various options resulted in 47 implementations for each taxon.

Results Species richness of the two taxonomic groups was predicted with good accuracy overall, and in most cases bS-SDM did not produce a biased prediction exceeding the actual number of species in each unit. In the prediction of community composition bS-SDM often also yielded the best evaluation score. In the case of poor performance of bS-SDM (i.e. when bS-SDM overestimated the prediction of richness) the SESAM framework improved predictions of species composition.

Main conclusions Our results differed from previous findings using community-level models. First, we show that overprediction of richness by bS-SDM is not a general rule, thus highlighting the relevance of producing good individual SDMs to capture the ecological filters that are important for the assembly process. Second, we confirm the potential of SESAM when richness is overpredicted by bS-SDM; limiting the number of species for each unit and applying biotic rules (here using the ranking of SDM probabilities) can improve predictions of species composition.

\section{Keywords}

Biotic rules, co-occurrence analysis, macroecological models, SESAM framework, stacked species distribution models, thresholding. 


\section{INTRODUCTION}

Understanding and predicting the attributes of biological communities across the landscape is a great challenge for ecologists. Many modelling approaches have been proposed to date for predicting communities (e.g. Ferrier \& Guisan, 2006; Shipley et al., 2006; Mokany et al., 2012), but so far one of the most widely used methods is to model and then stack the responses of individual species (i.e. summing predicted probabilities from individual species distribution models, S-SDMs) (e.g. Aranda \& Lobo, 2011; Dubuis et al., 2011; Faleiro et al., 2013; Pottier et al., 2013). This method has the advantage of being easy to apply, and once the predictions have been transformed to the binary scale (binary stacked species distribution model, bS-SDM), it can also predict the assemblage composition. However, the stacking of binary SDMs has shown a general tendency to exceed the actual number of species found in each unit (richness overprediction) when considering different taxonomic groups, areas and scales (e.g. Guisan \& Rahbek, 2011; Pineda \& Lobo, 2012; Calabrese et al., 2014; Cord et al., 2014; D’Amen et al., 2015). To explain this overprediction, Calabrese et al. (2014) suggested that the application of thresholds (i.e. choosing a particular value to transform a continuous probability distribution into a binary one) before stacking species predictions would cause incorrect overestimates of richness. Guisan \& Rahbek (2011) proposed the biological hypothesis that bS-SDM could overpredict richness because not all species for which the environment would be predicted as suitable co-occur in a given site due to a limited environmental carrying capacity and the exclusion of some species by biotic interactions.

Guisan \& Rahbek (2011) more generally proposed a novel modelling framework called 'spatially explicit species assemblage modelling' (SESAM) to integrate the different drivers of the assembly process that determine the final community. SESAM is applied in four steps in each unit:

1. Definition of a list of species that, due to historical and current dispersal limitations, could have reached the local unit (local species pool, SSP).

2. Application of a habitat filter based on the ecological niche model of each species. We use here the term habitat to identify the variety of conditions and resources that may satisfy the requirements of a species (Begon et al., 1996), thus including factors related to climate, topography and environment as in widespread use in the field of spatial predictive modelling (Guisan \& Zimmermann, 2000).

3. Definition of macroecological constraints that set a limit on the number of species that can theoretically co-occur in a given unit based on the space and/or resources available (macroecological model, MEM).

4. Application of biotic rules to select, from those species predicted from Step 2, the ones that can actually assemble to form the considered community. These ecological assembly rules (EARs) are defined as 'ecological restrictions on the observed patterns of species assemblages that are based on one or more other species or groups of species' (Guisan \& Rahbek, 2011) (Fig. 1, top right box).
The steps of SESAM can be put into practice with different approaches to predict species and biodiversity distributions. Only a single test of the implementation of SESAM has been conducted to date on plants (D'Amen et al., 2015). Plant species are known to strongly compete for space (le Roux et al., 2014), and therefore the SESAM framework was expected to produce better predictions of community richness and composition than models based solely on abiotic environmental filtering. D'Amen et al. (2015) tested different implementations of SESAM and were able to improve the prediction from bS-SDM by applying both a macroecological constraint to species richness and a simple biotic rule based on a ranking of the probability of the presence of the species in the sites (from best to least predicted). Departing from the results obtained in this former test on plant species, we aim here to spatially predict community richness and composition for two insect groups (butterflies and grasshoppers) in a mountain environment at high resolution. This is the first test of SESAM on animal assemblages: we evaluate different combinations of methods to implement Steps 2, 3 and 4, and we compare the performance of SESAM against the widely used bS-SDM (Fig. 1). We are also particularly interested in assessing whether constraints on species richness and assembly rules can be found within these groups, as these are often expected to compete to a much lesser extent than plant species.

Because the implementation of SESAM should be optimized for the study case, we fitted SDMs in Step 2 according to the ecology of the considered species; they are herbivores therefore we included proxies of plant resources as predictors (Araújo \& Luoto, 2007). This should, per se, limit the richness overprediction when SDMs are stacked to make a community prediction. Because we did not use just abiotic predictors, we produced a habitat pool sensu lato for the SESAM framework, though not a purely abiotic habitat pool, as in the original formulation. In producing both the bS-SDM and some of the SESAM implementations, the probability outcomes from SDM should be transformed into binary predictions. This is a critical step in individual species modelling, and it has been largely explored in the SDM literature (e.g. Liu et al., 2005), but less so in the context of community prediction (Pineda \& Lobo, 2009; Benito et al., 2013). To test the effect of using different thresholds in bS-SDM and SESAM, we ran all our analyses using five threshold maximization metrics, and we discuss the results obtained in community predictions (Step 2, Fig. 1).

The other novel test that we perform here is the inclusion of explicit ecological assembly rules (EARs) in the SESAM framework (Step 4, Fig. 1). Research on ecological assembly rules is a re-emerging field, but these rules have rarely been developed and applied to recompose communities from an environmentally filtered species pool (e.g. Cornwell \& Ackerly, 2009), and we know of no examples for insects. We developed new approaches to apply EARs considering only potential interactions within the taxonomic group (i.e. no trophic relations) on the basis of the spatial patterns of geographic overlap in the distributions of the species in the study area. We base the approaches on the assumption that processes structuring 
Figure 1 Schematic representation of the research questions in the test of spatially explicit species assemblage modelling (SESAM) (top left box), of the four steps in the framework (top right box) and of the alternative implementations in each step (bottom box).

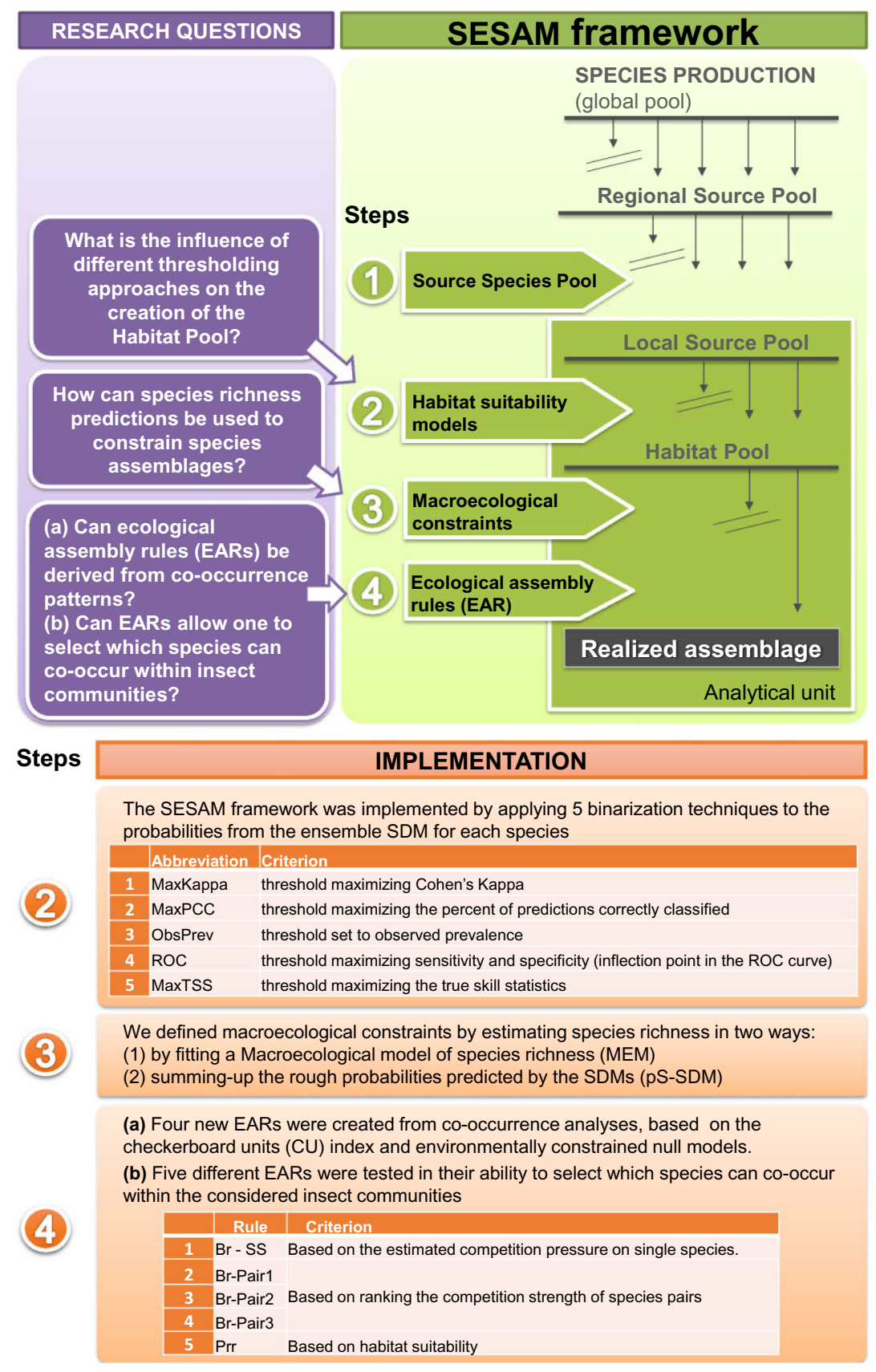

the community leave an imprint on the spatial distributions of species (e.g. Stone \& Roberts, 1990). Our goal was to assess whether identifiable pairwise interactions exist in these groups that can exclude species from an assemblage (e.g. 2000). However, the demonstration of significant non-randomness in the co-occurrence of species does not provide direct insights into the mechanisms that have generated the pattern (Connor \& Simberloff, 1979). To maximize the chance of distinguishing between the influence of environmental preferences and biotic interactions, we applied the approach proposed by Peres-Neto et al. (2001) to control for species habitat requirements during the creation of null communities. In particular we ask the following: (1) can EARs be derived from co-occurrence patterns and (2) are they useful for the fine-scale prediction of insect communities? The new EARs were integrated in SESAM and tested in terms of their success in improving community predictions (Fig. 1).

\section{METHODS}

\section{Community data and environmental variables}

The study area is located in the western Swiss Alps, Canton de Vaud, $\left(46^{\circ} 10^{\prime}-46^{\circ} 30^{\prime} \mathrm{N} ; 6^{\circ} 50^{\prime}-7^{\circ} 10^{\prime} \mathrm{E}\right)$ and covers approximately $700 \mathrm{~km}^{2}$, with a strong elevation gradient from 375 to $3210 \mathrm{~m}$ a.s.l. Sample sites were selected outside forested areas, 
according to a random stratified design based on elevation, slope and aspect. Samplings were performed between July and September in 2009, 2010 and 2012, during the hours when the insects were active and under good weather conditions. Butterflies were collected in 192 squares of $50 \mathrm{~m} \times 50 \mathrm{~m}$, ranging between 1000 and $3210 \mathrm{~m}$ a.s.1., while the sampling for grasshoppers was conducted over the whole elevational range for a total of 202 squares (see Pellissier et al., 2012, and Pradervand et al., 2013, for more information).

A total of 131 butterfly and 41 grasshopper species were collected. Due to modelling limitations, we only considered those species that had more than 20 occurrences: 77 butterflies and 20 grasshoppers. We considered four bioclimatic variables (solar radiation, summer temperature, annual degree-days and annual average number of frost days during the growing season), an index of vegetation productivity, i.e. normalized difference vegetation index (as proxies for trophic resources), and the distance to forest (see Appendix S1 in Supporting Information for descriptions of the variables and references). These variables were selected on the basis of insect ecology (e.g. Turner et al., 1987; Hawkins, 2003) and experiences from previous investigations for the same study systems (e.g. Pellissier et al., 2012; Pradervand et al., 2013). We verified that the correlation between pairs of variables was lower than 0.7 to avoid multicollinearity problems.

\section{Analytical steps}

To implement the SESAM framework, we adopted the four-step procedure as described in Guisan \& Rahbek (2011) (Fig. 1, top right box).

Step 1: Definition of the pool of source species

In the present study, due to the limited geographical extent and high resolution, all species in the regional source pool are considered equally probable.

\section{Step 2: Definition of the habitat pool}

The habitat pool was defined by fitting individual SDMs for all the species using the variables described above (Appendix S1). We modelled species distributions using four statistical techniques appropriate for fitting presence-absence SDMs, assuming a binomial distribution: a generalized linear model (GLM), a generalized additive model (GAM), a generalized boosted model (GBM) and random forest (RF). We used a repeated 10 split-sample procedure for the evaluation: each model was fitted using $70 \%$ of the data and evaluated using the remaining $30 \%$ by the area under the curve (AUC) of a receiver operating characteristics (ROC) plot (Fielding \& Bell, 1997). To obtain a final prediction, we applied an ensemble forecasting framework by averaging all single-model projections, weighted by their respective evaluation scores (Marmion et al., 2009). For those species with 50 occurrences or fewer, simple SDMs were fitted with all predictors, and then the consensus average was calculated. Species with between 20 and 50 occurrences were modelled with the Ensemble Bivariate approach (Lomba et al., 2010): we fitted individual models based on bivariate combinations of the selected predictors with the four modelling techniques mentioned above, and we created a consensus forecast from all the resulting models by a weighted mean of their predictions, with weights based on the AUC scores. The modelling analyses were performed in R 3.0.1 ( $\mathrm{R}$ Development Core Team, 2014) using the BIOMOD2 package (Thuiller et al., 2014). Probabilities from SDMs were converted into binary maps by applying five thresholding methods (Fig. 1; see Appendix S2 for references to threshold methods and Liu et al., 2005, for further description). Consequently, we produced five presence/absence predictions for each species and the same number of binary habitat pools and bS-SDMs for both orders of insects by summing the binary maps obtained for each species (Fig. 1).

\section{Step 3: Macroecological constraints}

We used two methods to define macroecological constraints. First, we summed the probabilities from SDMs (Dubuis et al., 2011) for the species, obtaining a prediction of richness for each unit (pS-SDM). Second, we modelled the observed species richness separately for the two groups using a GLM, GAM, GBM and RF as a function of six environmental predictors (Appendix S1) with a Poisson link function. The observed species richness (SR) was calculated as the count of all species belonging to the two insect groups that occurred in more than 20 plots. Each model was run 10 times with a repeated splitsample procedure: models were fitted on the $70 \%$ data partition, and the other $30 \%$ was used for independent evaluation, i.e. the Spearman rank correlation between observed and predicted SR. Finally, we calibrated the models with $100 \%$ of the data so as to avoid biases associated with subsampling (Araújo et al., 2005). An ensemble SR estimate for each group (MEMs models) was obtained by averaging the predictions from full models run with the four different techniques weighted by their mean correlation scores.

\section{Step 4: Creation and integration of ecological assembly rules}

We implemented the SESAM framework by applying different rules. First, we applied the probability ranking rule (PRR) proposed by D'Amen et al. (2015): we selected a number of species equal to the prediction of species richness on the basis of decreasing probability of presence calculated by the SDMs. This rule assumes that the predicted probabilities calculated by SDMs are a proxy for the competitive strength of species. We therefore hypothesize that a species that has a higher probability of presence will have a competitive advantage over another species with a lower predicted probability by being closest to its optimal performance in the considered unit.

In addition, we created new EARs on the basis of the spatial patterns of co-occurrence in species distributions. We considered the number of species pairs that never co-occur, forming 
'checkerboard' distributions ('checkerboard units', CU), as an indication of pairwise species interaction (Diamond, 1975). We evaluated the significance of the observed CU indices with environmentally weighted null models (Peres-Neto et al., 2001), correcting for the false discovery rate with an empirical Bayes approach (Bayes CL criterion; Gotelli \& Ulrich, 2010). To allow comparisons across species pairs and insect groups, we scaled the results in units of standard deviations (Gurevitch et al., 1992). On the basis of the CU of species pairs and their predicted significance level, we developed two sets of EARs (biotic rules, $\mathrm{Br}$ ) based either on estimating a 'competition pressure' for a single species (Br-SS) or on the interaction strength for species pairs (Br-Pair). We tested three different implementations of the Br-Pair, where we progressively relaxed the limitation of matching the number of species in each unit to the macroecological constraint. All these rules are applied on the five habitat pools for each site obtained in Step 2 (see Appendix S3 for details).

\section{Evaluation}

We evaluated the richness predictions by calculating the correlation between the observed species richness, the mean absolute error (MAE) and the prediction bias (ME, mean of the error) (Steinmann et al., 2009). To evaluate the community predictions derived from the application of the SESAM framework and bS-SDMs, we calculated the species richness error (predicted SR - observed SR, expressed as a number of species), and we used two metrics based on a confusion matrix totalling the number species predictions that were true positives (TP), true negatives (TN), false positives (FP) and false negatives (FN) (Fielding \& Bell, 1997) in any cell:

$$
\text { prediction success }=\frac{\mathrm{TP}+\mathrm{TN}}{\mathrm{SP}}
$$

Sørensen index $=\frac{2 \mathrm{TP}}{2 \mathrm{TP}+\mathrm{FN}+\mathrm{FP}}$.
The prediction success evaluates the ability to filter out absent species and to predict the occurrences of observed species from the entire regional species pool, while the Sørensen index, a widely used metric of community similarity, does not account for the true absences in the calculation.

\section{RESULTS}

\section{Modelling results}

The ensemble prediction from the individual SDMs for both insect groups had very high AUC values, with means of 0.969 for grasshoppers and 0.965 for butterflies (Appendix S4). Predictions of total richness calculated using different approaches were all very good, showing a Pearson correlation higher than 0.7 for butterflies and 0.85 for grasshoppers (Table 1 ). In addition, the mean error (ME) and the mean absolute error (MAE) indices showed a strong model performance in predicting richness (Table 1). The highest ME and MAE were obtained by the 'bSSDM ObsPrev' model for both taxa, due to a moderate degree of overestimation (approximately 20\% and 15\% for butterflies and grasshoppers, respectively) (Table 1, Appendix S2).

\section{Co-occurrence analysis}

The insect communities were analysed for the co-occurrence pattern in the study area. Overall, the butterfly communities showed a strong aggregated pattern, with the observed $C$-score being significantly lower than the mean of the simulated $C$-score indices $\left(C\right.$-score $_{\text {obs }}=817.95, \quad C$ - score $_{\text {Sim }}=890.01$, $P$-value $\left(\mathrm{Obs}_{\mathrm{s}<\mathrm{Sim})}<0.0001\right)$. A weaker aggregated pattern was observed in the grasshopper communities: the observed $C$-score was significantly lower than the mean of the simulated $C$-score indices $\left(C\right.$-score $_{\mathrm{obs}}=665.87, \quad C$-score $_{\mathrm{Sim}}=720.75$, $P$-value $\left._{(\mathrm{Obs}<\mathrm{Sim})}=0.009\right) \quad($ Appendix S5). With regard to the pairwise interactions, after the application of the Bayes CL criterion to control for false discovery rates, we identified 229 out

Table 1 Evaluation scores for predictions of total richness according to the following evaluation metrics: mean of the error (ME), the mean absolute error (MAE), and the Pearson correlation with the observed species richness ( $r$ Pearson).

\begin{tabular}{|c|c|c|c|c|c|c|}
\hline \multirow[b]{2}{*}{ Modelling approach } & \multicolumn{2}{|l|}{ ME } & \multicolumn{2}{|l|}{ MAE } & \multicolumn{2}{|l|}{ r Pearson } \\
\hline & Butterflies & Grasshoppers & Butterflies & Grasshoppers & Butterflies & Grasshoppers \\
\hline pS-SDM & 1.014 & 0.014 & 4.656 & 1.222 & 0.796 & 0.881 \\
\hline MEM & -3.771 & -0.693 & 5.625 & 1.307 & 0.758 & 0.879 \\
\hline bS-SDM ROC & 3.661 & 0.728 & 4.484 & 1.411 & 0.874 & 0.862 \\
\hline bS-SDM MaxKappa & -0.146 & -0.691 & 3.01 & 0.941 & 0.899 & 0.914 \\
\hline bS-SDM MaxTSS & 4.406 & 0.926 & 4.906 & 1.413 & 0.873 & 0.895 \\
\hline bS-SDM ObsPrev & 15.832 & 3.193 & 15.833 & 3.322 & 0.712 & 0.851 \\
\hline bS-SDM MaxPCC & -2.05 & -0.381 & 3.333 & 0.945 & 0.904 & 0.919 \\
\hline
\end{tabular}

Abbreviations: pS-SDM, sum of probabilities from single species distribution models; MEM, macroecological models; ROC, threshold where the ROC curve makes its closest approach to (0,1); MaxKappa, threshold that maximizes Kappa; MaxTSS, threshold set at (sensitivity + specificity - 1); ObsPrev, threshold set to the observed prevalence; MaxPCC, threshold that results in the maximum percentage of correctly classified. 

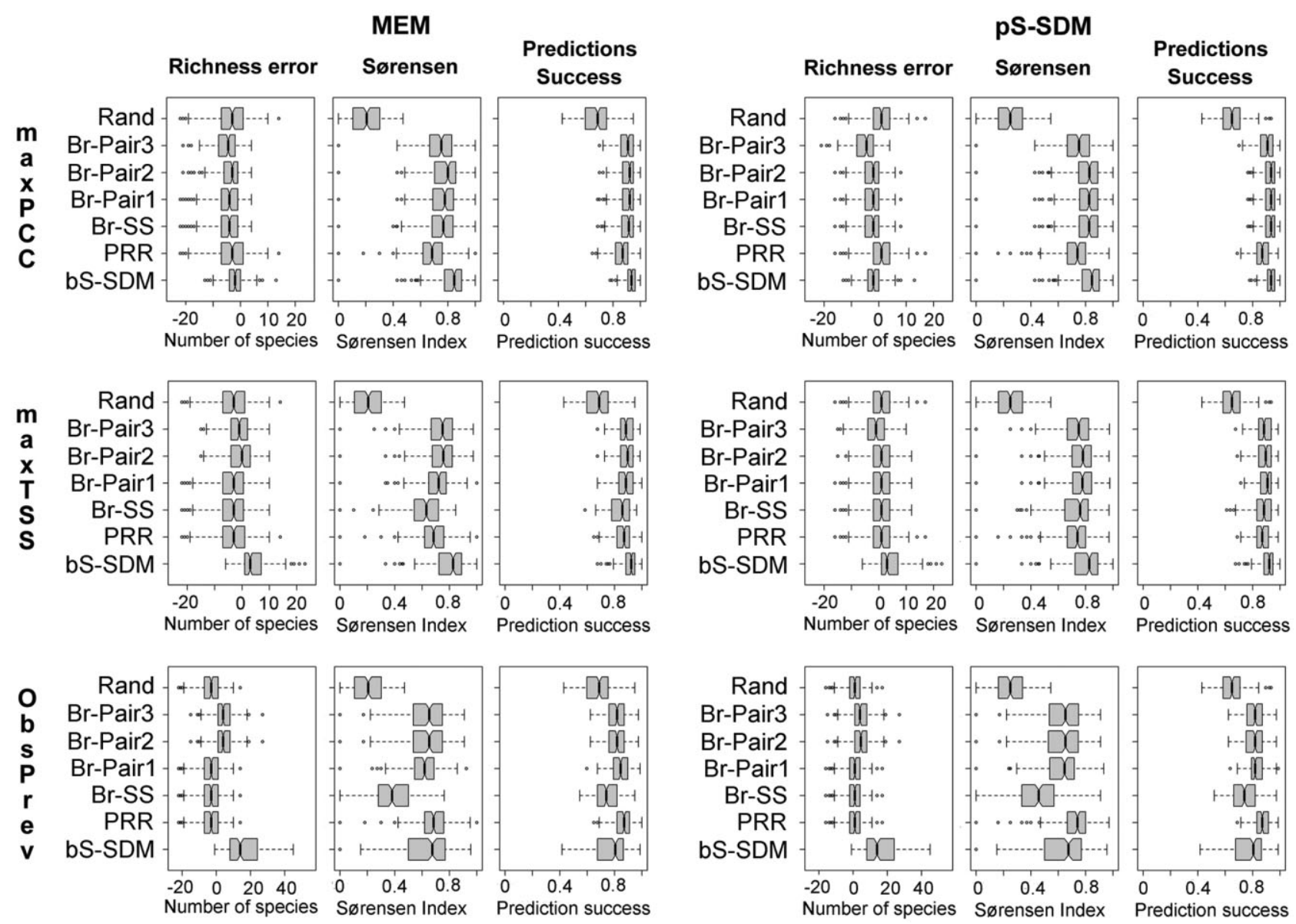

Figure 2 Boxplots representing the richness error, the Sørensen index and the prediction success of species richness and composition for the prediction obtained from the sum of binary individual species distribution models (bS-SDM), the differential implementation of the spatially explicit species assemblage modelling (SESAM) framework in Steps 2 (here we show results from the application of three thresholds), 3 (two richness models) and 4 (five biotic rules), and a random sorting of species to match the value from the relevant richness prediction (Rand). Abbreviations: pS-SDM, sum of probabilities from single species distribution models; MEM, macroecological models; MaxTSS, threshold set at (sensitivity + specificity - 1); ObsPrev, threshold set to the observed prevalence; MaxPCC, threshold that results in the maximum percentage of correctly classified sites; Br-SS, biotic rule based on competition pressure on single species; Br-Pair1, biotic rule based on the competition strength of species pairs without considering a thresholding $P$-value; Br-Pair2, biotic rule based on the competition strength of species pairs showing a significant segregation pattern after the application of the Bayesian CL correction; Br-Pair3, biotic rule that always removes one of the two significantly competing species, if any are present in the unit; PRR, probability ranking rule, based on the probability of the presence of the species in the unit.

2927 pairs of butterfly species and 22 out 190 pairs of grasshopper species with significant segregated patterns (Appendix S6).

\section{Overall performance of community predictions}

The implementation of bS-SDMs and of the SESAM framework, considering all the combinations of techniques proposed for implementing the three steps, leads to 47 different community predictions for both insect taxa (Fig. 1). For butterflies, in the cases of overprediction from the binary habitat pool, the application of the biotic rules caused a reduction in the richness error. As expected, Br-Pair3 most often produced a bias in the prediction toward underestimation of the number of species (richness underprediction), even if the mean error was always fewer than six species. In the cases of underprediction from the binary habitat pool, the application of the biotic rules produced a larger error of underestimation (Fig. 2). In the grasshopper community, which contains fewer species, the differences measured as richness error are very small; the highest error is the overprediction (mean error of three species) by the simple bS-SDM when the applied threshold was ObsPrev (Appendix S2). Almost all the other SESAM implementations varied between +1 and -1 (Fig. 2).

We obtained very positive results in composition predictions according to both evaluation metrics and for both groups. Generally, the community composition was better estimated by the simple bS-SDM than by the application of SESAM, except for 
Table 2 Performance in predicting species composition (mean Sørensen index) of the sum of binary individual species distribution models (bS-SDM) implemented with five different thresholds (left column) and each combination of the spatially explicit species assemblage modelling (SESAM) framework in Steps 2 (five thresholds), 3 (two richness models) and 4 (five biotic rules). On the colour scale, darker tones highlight increasingly good predictive performances (higher values of the index).

\begin{tabular}{|c|c|c|c|c|c|c|c|c|}
\hline \multirow[b]{3}{*}{ bS-SDM } & \multicolumn{7}{|c|}{ SESAM implementation } & \\
\hline & \multirow[b]{2}{*}{ Thresholds } & \multirow[b]{2}{*}{ Richness models } & \multicolumn{5}{|c|}{ Biotic rules } & \\
\hline & & & $\mathrm{Br}-\mathrm{SS}$ & Br-Pair1 & Br-Pair2 & Br-Pair3 & PRR & \\
\hline 0.827 & MaxTSS & pS-SDM & 0.760 & 0.777 & 0.780 & 0.750 & 0.741 & Butterflies \\
\hline 0.817 & ROC & pS-SDM & 0.758 & 0.789 & 0.780 & 0.750 & 0.741 & \multirow{19}{*}{ Grasshoppers } \\
\hline 0.846 & MaxPCC & pS-SDM & 0.826 & 0.826 & 0.828 & 0.752 & 0.741 & \\
\hline 0.847 & MaxKappa & pS-SDM & 0.812 & 0.819 & 0.816 & 0.758 & 0.741 & \\
\hline \multirow[t]{6}{*}{0.675} & ObsPrev & pS-SDM & 0.457 & 0.646 & 0.653 & 0.653 & 0.741 & \\
\hline & MaxTSS & MEM & 0.632 & 0.722 & 0.756 & 0.750 & 0.684 & \\
\hline & ROC & MEM & 0.667 & 0.727 & 0.750 & 0.750 & 0.684 & \\
\hline & MaxPCC & MEM & 0.767 & 0.778 & 0.800 & 0.752 & 0.684 & \\
\hline & MaxKappa & MEM & 0.737 & 0.763 & 0.777 & 0.758 & 0.684 & \\
\hline & ObsPrev & MEM & 0.381 & 0.619 & 0.653 & 0.653 & 0.684 & \\
\hline 0.850 & MaxTSS & pS-SDM & 0.800 & 0.824 & 0.824 & 0.824 & 0.800 & \\
\hline 0.769 & ROC & pS-SDM & 0.727 & 0.769 & 0.769 & 0.769 & 0.800 & \\
\hline 0.889 & MaxPCC & pS-SDM & 0.842 & 0.857 & 0.857 & 0.842 & 0.800 & \\
\hline 0.890 & MaxKappa & pS-SDM & 0.857 & 0.857 & 0.875 & 0.857 & 0.800 & \\
\hline \multirow[t]{6}{*}{0.714} & ObsPrev & pS-SDM & 0.667 & 0.750 & 0.756 & 0.756 & 0.800 & \\
\hline & MaxTSS & MEM & 0.750 & 0.800 & 0.824 & 0.824 & 0.769 & \\
\hline & ROC & MEM & 0.667 & 0.714 & 0.769 & 0.769 & 0.769 & \\
\hline & MaxPCC & MEM & 0.800 & 0.800 & 0.857 & 0.842 & 0.769 & \\
\hline & MaxKappa & MEM & 0.800 & 0.800 & 0.875 & 0.857 & 0.769 & \\
\hline & ObsPrev & MEM & 0.667 & 0.750 & 0.756 & 0.756 & 0.769 & \\
\hline
\end{tabular}

Abbreviations: pS-SDM, sum of probabilities from single species distribution models; MEM, macroecological models; ROC, threshold where the ROC curve makes its closest approach to (0,1); MaxKappa, threshold that maximizes Kappa; MaxTSS, threshold set at (sensitivity + specificity - 1); ObsPrev, threshold set to the observed prevalence; MaxPCC, threshold that results in the maximum percentage of correctly classified sites; Br-SS, biotic rule based on the competition pressure on a single species; Br-Pair1, biotic rule based on the competition strength of species pairs without considering a thresholding $P$-value; Br-Pair2, biotic rule based on the competition strength of species pairs showing a significant segregation pattern after the application of the Bayesian CL correction; Br-Pair3, biotic rule that always removes one of the two significantly competing species, if any are present in the unit; PRR, probability ranking rule, based on the probability of the presence of the species in the unit.

the case of the ObsPrev threshold (Appendix S2). In this latter case, the SESAM framework, when implemented in Step 4 with the PRR, produced the best community prediction in term of species composition (Table 2). The use of random species sorting to match the richness prediction in Step 3 always produced the worst results (mean Sørensen index $<0.5$ ). In particular, the community of butterflies was best predicted by the simple bS-SDM when we applied the thresholds maxPCC or maxK (Appendix S2) in Step 2 (mean Søresen index $=0.85$ ). We obtained the poorest SESAM prediction when applying the combination of the ObsPrev threshold to produce the habitat pool in Step 2, the MEM richness prediction in Step 3 and the biotic rule Br-SS in Step 4 (mean Sørensen index =0.38) (cf. Fig. 1 for the sequence of steps). All the other SESAM predictions had a mean Sørensen index $>0.6$. For grasshoppers, the predictions reached higher mean Sørensen indices with a similar pattern of performance; the poorest prediction was achieved by applying the Br-SS (mean Sørensen index $=0.66$ ), while the highest value of the index was obtained by predictions from the simple bS-SDM when the applied thresholds were maxPCC or maxK (mean Sørensen index =0.89) (Table 2, Appendix S2). The mean prediction success index was higher than 0.8 for all predictions in both groups. Results of differential implementations of the single steps can be found in Appendix S7.

\section{DISCUSSION}

Our capacity to understand the process of species assemblage in real, multispecies communities is still limited, and several options exist to enhance it (e.g. Laughlin et al., 2012; Mokany et al., 2012; Fernandes et al., 2013). Here we tested a proposed solution, i.e. the SESAM framework, to spatially predict community properties (e.g. richness, composition) by integrating different drivers of the assembly process in a unique workflow. Our results show an interesting picture that diverges in several aspects from previous findings but confirms the utility of the application of the SESAM framework in some circumstances. First, our community-level models predicted, with good overall 
accuracy, the species composition of the two insect groups. Previous studies analysing different taxonomic assemblages were not able to produce such accurate predictions of species composition (e.g. Aranda \& Lobo, 2011; Pottier et al., 2013; D'Amen et al., 2015). Species richness predictions also yielded high significant correlation scores with the observed richness that did not differ between methods as expected (macroecological model or summing the predicted probabilities from SDMs) (e.g. Calabrese et al., 2014; D'Amen et al., 2015; Distler et al., 2015). Remarkably, a good richness prediction was also obtained from the bS-SDM models. The error in predicting the number of species in different units was generally low; in only one case was a moderate degree of overprediction observed, when applying a particular thresholding method to create binary predictions (ObsPrev; i.e. the threshold where the predicted prevalence is equal to the observed prevalence). This result is quite different from previous findings, which all showed an overall tendency of bS-SDMs to overpredict species richness (Feria \& Peterson, 2002; Dubuis et al., 2011; Calabrese et al., 2014; D'Amen et al., 2015). However, this comparison should be taken with caution, because the evaluation approach used (e.g. external or internal validation) can differ among studies.

Our results show that richness overprediction by bS-SDM is not a rule. This novel finding can first be explained by the high quality of the individual SDMs. Following a biological explanation, the choice of the environmental variables is likely to have influenced the quality of our predictions. The importance of considering trophic resources in SDMs for modelling herbivorous insects, as we did, has already been shown (e.g. Araújo \& Luoto, 2007). As expected, the inclusion of bionomic (i.e. resource-related) variables that are ecologically relevant to the target species at this scale of analysis provided a greater approximation of the species realized niche (the Eltonian component of the niche; Soberon \& Nakamura, 2009) already limiting the richness overprediction when SDMs are stacked to create a community prediction. Our SDMs probably captured important ecological filters that restrict the composition of local species pools derived from the regional pool (Keddy, 1992), while this was not possible when only using climatic predictors. This explanation is supported by a previous study with the same butterfly dataset that produced less accurate community predictions by stacking SDMs fitted with climatic variables (Pellissier et al., 2012). More generally, the improvement in bS-SDM predictions related to the inclusion of additional variables in the individual models has also been reported recently for tree species in Mexico (Cord et al., 2014). Finally, the low degree of competition detected in the insect assemblages further facilitated the possibility of correctly modelling the community structure by applying an environmental filter that accounts for plant resources.

From a methodological point of view, the application of the ensemble of bivariate models (Lomba et al., 2010) is likely to have improved our predictive ability. This method avoids overfitting without losing explanatory power by averaging many small models (Breiner et al., 2015). Consequently we were able to model more species than with traditional modelling approaches, including those with a low number of presences in which they are usually ignored, and to use the number of predictors needed to capture all dimensions of a species' niche. Calabrese et al. (2014) suggested that thresholding before stacking SDMs would generally cause the statistical bias towards overprediction in bS-SDMs. Even following this purely statistical explanation, the very good quality of insect SDMs can explain the low error in our richness predictions: choosing a value to transform continuous predictions in presence and absence maps would produce a higher error when stacking models with low accuracy. Another factor potentially related to richness overprediction by bS-SDM could be the community dimension: the greater the number of species, the higher the potential biases that can accumulate in the final community prediction. To our knowledge, this aspect has never been extensively explored and could help explain some of the observed patterns. On the other hand, richness overprediction has also been observed in small and medium-sized datasets by Calabrese et al. (2014) (24 species in the Wadden Sea Macrobenthos dataset, 79 species in the Barents Sea trawls), which had dimensions comparable to those that we analysed.

Among the modelling approaches tested, overall community predictions by bS-SDM outperformed those obtained by different implementations of SESAM. Thus, our results suggest that, with high accuracy from SDMs, the best community prediction simply matches the binary habitat pool, obtained in Step 2 of SESAM, while the implementation of the other steps seems unnecessary. On the contrary, in the case of richness overprediction by bS-SDM the application of the whole SESAM framework improved the community prediction by setting a macroecological constraint limiting the number of co-existing species (Step 3), coupled with the implementation of biotic rules to identify the most probable ones in the unit (Step 4). Given these conditions, the PRR produced the best community predictions, i.e. selecting single species from the pool predicted by SDMs by decreasing order of predicted probability until the predicted richness is reached. This result is in accordance with a previous test of the PRR in the SESAM framework for a plant community in the same study area (D'Amen et al., 2015).

We acknowledge that the assumption of greater competitive power implicit in the PRR is an over-simplification and does not represent the full complexity of biotic interactions. Here, however, the PRR was not intended to represent all possible interspecific interactions in the insect communities considered. Developing formal assembly rules for a large number of species, as modelled in this study, would require the setting of large experiments coupled with physiological measurements, which has not been attempted so far. Research on ecological assembly rules is seeking to identify solutions to reduce this complexity at a lower cost (e.g. Gilman et al., 2010; Kissling et al., 2012), and the PRR can be seen as a first attempt at this. The good performance of the PRR can be also interpreted from a more technical point of view. For instance, as pointed out by D'Amen et al. (2015), the good performance of the PRR can be related to the fact that the same species with the lower probabilities of 
occurrence, i.e. the ones removed by the rule, are also those most likely to be overpredicted by bS-SDMs.

The new biotic rules based on the co-occurrence analysis improved the overprediction by bS-SDM. In particular, the best predictions were obtained by Br-Pair 2: the strengths of this biotic rule are that it considers only those species pairs that show a significant segregation pattern and allows for the retention of the binary habitat pool prediction if not enough competing pairs are present in the unit to reach the macroecological constraint. The approach of using a co-occurrence analysis to define potentially exclusive species pairs to use as biotic rules in SESAM was interesting and worth testing, but did not improve the predicted compositions for the considered insect communities for all conditions. A reason for this result could be the overall aggregation pattern detected in both communities: the low-competitive structure could be linked to the determination of low intra-guild interactions by the geographical properties of the study area. In mountainous environments, abiotic conditions are expected to become the main determinants of species assemblages toward higherelevation areas (e.g. Pottier et al., 2013). Moreover, it has been shown that the degree of community patterning varies among taxonomic groups: homeotherms more often have a nonrandom community pattern than heterotherms (like our study groups), which is probably linked to their different physiological requirements (Gotelli \& McCabe, 2002). The checkerboard pattern can be also due to affinities for non-overlapping habitats and not to competitive interactions: our co-occurrence analysis coupled with environmentally weighted null models should have increased the confidence that species pairs with a segregated distribution for competitive reasons are correctly identified. Moreover, we used presence/absence data: thus, the co-occurrence analyses can indicate interactive pairs whose interactions lead to competitive exclusion, but we were not able to consider weaker forms of competition potentially relevant to our communities.

\section{CONCLUDING REMARKS}

In this paper we have explored different methods for spatial prediction of insect communities. In particular, we implemented one of the most recently proposed solutions - the SESAM framework - for the first time for animal communities. Using our results, we have derived useful new insights into the application of the framework and on how to model communities in general. First, we highlighted the importance of producing good SDMs to reconstruct communities. If SDMs are fitted in a biased manner or miss the relevant predictors representing the environmental filter they offer little information about the ecological potential of the species, and when applying SESAM the framework will be similarly biased. Second, according to our current results and those from a previous test of SESAM on plant communities, the benefit of applying SESAM is higher when the SDMs alone are not able to depict the community structure, and a symptom of this is the overprediction of richness by bS-SDM. On the contrary, when this bias is not observed, the application of all steps of SESAM cannot further improve the prediction of community composition. Third, in relation to the biotic rules in SESAM, we can confirm the utility of the previously proposed PRR (D'Amen et al., 2015) for improving community predictions if an overprediction in the binary habitat pool is detected. Finally, considering the community characteristics, we cannot reach a definitive conclusion about the proposed biotic rules based on co-occurrence, but we have identified the best performing one so far. Further tests on species assemblages structured by competition could help clarify the utility of biotic rules based on co-occurrence patterns. However, a preliminary analysis of co-occurrence in the study area could help decide if the community model would benefit from the inclusion of biotic rules. Other investigations of biotic interactions could complement or substitute for this co-occurrence analysis, while also considering population densities when data are available.

\section{ACKNOWLEDGEMENTS}

We are grateful to the numerous people who contributed to data collection, especially Loïc Pellisser, who also gave useful suggestions for the final writing of the manuscript. We also thank $\mathrm{N}$. Gotelli for his insights into the statistics of co-occurrence analysis and the application of the false discovery rate. M.D. was supported by a Marie Curie Intra-European Fellowship (FP7PEOPLE-2012-IEF, SESAM-ZOOL 327987).

\section{REFERENCES}

Aranda, S.C. \& Lobo, J.M. (2011) How well does presence-onlybased species distribution modelling predict assemblage diversity? A case study of the Tenerife flora. Ecography, 34, 31-38.

Araújo, M.B. \& Luoto, M. (2007) The importance of biotic interactions for modelling species distributions under climate change. Global Ecology and Biogeography, 16, 743-753.

Araújo, M.B., Pearson, R.G., Thuiller, W. \& Erhard, M. (2005) Validation of species-climate impact models under climate change. Global Change Biology, 11, 1504-1513.

Begon, M., Harper, J.L. \& Townsend, C.R. (1996) Ecology: individuals, populations and communities, 3rd edn. Blackwell Science, Oxford.

Benito, B.M., Cayuela, L., Albuquerque, F.S. \& O'Hara, R.B. (2013) The impact of modelling choices in the predictive performance of richness maps derived from speciesdistribution models: guidelines to build better diversity models. Methods in Ecology and Evolution, 4, 327-335.

Breiner, F.T., Guisan, A., Bergamini, A. \& Nobis, M.P. (2015) Overcoming limitations of modelling rare species by using ensembles of small models. Methods in Ecology and Evolution, doi:10.1111/2041-210X.12403.

Calabrese, J.M., Certain, G., Kraan, C. \& Dormann, C.F. (2014) Stacking species distribution models and adjusting bias by linking them to macroecological models. Global Ecology and Biogeography, 23, 99-112. 
Connor, E.F. \& Simberloff, D. (1979) The assembly of species communities: chance or competition? Ecology, 60, 1132-1140.

Cord, A.F., Klein, D., Gernandt, D.S., de la Rosa, J.A.P., Dech, S. \& McGeoch, M. (2014) Remote sensing data can improve predictions of species richness by stacked species distribution models: a case study for Mexican pines. Journal of Biogeography, 41, 736-748.

Cornwell, W.K. \& Ackerly, D.D. (2009) Community assembly and shifts in plant trait distributions across an environmental gradient in coastal California. Ecological Monographs, 79, 109126.

D’Amen, M., Dubuis, A., Fernandes, R.F., Pottier, J., Pellisser, L. \& Guisan, A. (2015) Using species richness and functional traits predictions to constrain assemblage predictions from stacked species distribution models. Journal of Biogeography, 42, 1255-1266.

Diamond, J.M. (1975) Assembly of species communities. Ecology and evolution of communities (ed. by M.L. Cody and J.M. Diamond), pp. 342-444. Harvard University Press, Cambridge, MA.

Distler, T., Schuetz, J.G., Velásquez-Tibatá, J., Langham, G.M. \& Ladle, R. (2015) Stacked species distribution models and macroecological models provide congruent projections of avian species richness under climate change. Journal of Biogeography, 42, 976-988.

Dubuis, A., Pottier, J., Rion, V., Pellissier, L., Theurillat, J.P. \& Guisan, A. (2011) Predicting spatial patterns of plant species richness: a comparison of direct macroecological and species stacking modelling approaches. Diversity and Distributions, 17, 1122-1131.

Faleiro, F.V., Machado, R.B. \& Loyola, R.D. (2013) Defining spatial conservation priorities in the face of land-use and climate change. Biological Conservation, 158, 248-257.

Feria, A.T.P. \& Peterson, A.T. (2002) Prediction of bird community composition based on point-occurrence data and inferential algorithms: a valuable tool in biodiversity assessments. Diversity and Distributions, 8, 49-56.

Fernandes, J.A., Cheung, W.W., Jennings, S., Butenschon, M., De Mora, L., Frolicher, T.L. \& Grant, A. (2013) Modelling the effects of climate change on the distribution and production of marine fishes: accounting for trophic interactions in a dynamic bioclimate envelope model. Global Change Biology, 19, 2596-2607.

Ferrier, S. \& Guisan, A. (2006) Spatial modelling of biodiversity at the community level. Journal of Applied Ecology, 43, 393404.

Fielding, A.H. \& Bell, J.F. (1997) A review of methods for the assessment of prediction errors in conservation presence/absence models. Environmental Conservation, 24, $38-49$.

Gilman, S.E., Urban, M.C., Tewksbury, J., Gilchrist, G.W. \& Holt, R.D. (2010) A framework for community interactions under climate change. Trends in Ecology and Evolution, 25, 325331.

Gotelli, N.J. (2000) Null model analysis of species co-occurrence patterns. Ecology, 81, 2606-2621.
Gotelli, N.J. \& McCabe, D.J. (2002) Species co-occurrence: a meta-analysis of Diamond's assembly rules model. Ecology, 83, 2091-2096.

Gotelli, N.J. \& Ulrich, W. (2010) The empirical Bayes approach as a tool to identify non-random species associations. Oecologia, 162, 463-477.

Guisan, A. \& Rahbek, C. (2011) SESAM-a new framework integrating macroecological and species distribution models for predicting spatio-temporal patterns of species assemblages. Journal of Biogeography, 38, 1433-1444.

Guisan, A. \& Zimmermann, N.E. (2000) Predictive habitat distribution models in ecology. Ecological Modelling, 135, 147186.

Gurevitch, J., Morrow, L.L., Wallace, A. \& Walsh, J.S. (1992) A meta-analysis of field experiments on competition. The American Naturalist, 140, 539-572.

Hawkins, B.A. (2003) Water-energy balance and the geographic pattern of species richness of western Palearctic butterflies. Ecological Entomology, 41, 678-686.

Keddy, P. (1992) Assembly and response rules: two goals for predictive community ecology. Journal of Vegetation Science, 3, 157-164.

Kissling, W.D., Dormann, C.F., Groeneveld, J., Hickler, T., Kuhn, I., McInerny, G.J., Montoya, J.M., Römermann, C., Schiffers, K., Schurr, F.M., Singer, A., Svenning, J.-C., Zimmermann, N.E. \& O'Hara, R.B. (2012) Towards novel approaches to modelling biotic interactions in multispecies assemblages at large spatial extents. Journal of Biogeography, 39, 21632178.

le Roux, P.C., Pellissier, L., Wisz, M.S. \& Luoto, M. (2014) Incorporating dominant species as proxies for biotic interactions strengthens plant community models. Journal of Ecology, 102, 767-775.

Laughlin, D.C., Joshi, C., van Bodegom, P.M., Bastow, Z.A. \& Fule, P.Z. (2012) A predictive model of community assembly that incorporates intraspecific trait variation. Ecology Letters, 15, 1291-1299.

Liu, C., Berry, P., Dawson, T. \& Pearson, R. (2005) Selecting thresholds of occurrence in the prediction of species distributions. Ecography, 28, 385-393.

Lomba, A., Pellissier, L., Randin, C., Vicente, J., Moreira, F., Honrado, J. \& Guisan, A. (2010) Overcoming the rare species modelling paradox: a novel hierarchical framework applied to an Iberian endemic plant. Biological Conservation, 143, 26472657.

Marmion, M., Parviainen, M., Luoto, M., Heikkinen, R.K. \& Thuiller, W. (2009) Evaluation of consensus methods in predictive species distribution modelling. Diversity and Distributions, 15, 59-69.

Mokany, K., Harwood, T.D., Williams, K.J. \& Ferrier, S. (2012) Dynamic macroecology and the future for biodiversity. Global Change Biology, 18, 3149-3159.

Pellissier, L., Pradervand, J.-N., Pottier, J., Dubuis, A., Maiorano, L. \& Guisan, A. (2012) Climate-based empirical models show biased predictions of butterfly communities along environmental gradients. Ecography, 35, 684-692. 
Peres-Neto, P.R., Olden, J.D. \& Jackson, D.A. (2001) Environmentally constrained null models: site suitability as occupancy criterion. Oikos, 93, 110-120.

Pineda, E. \& Lobo, J.M. (2009) Assessing the accuracy of species distribution models to predict amphibian species richness patterns. Journal of Animal Ecology, 78, 182-190.

Pineda, E. \& Lobo, J.M. (2012) The performance of range maps and species distribution models representing the geographic variation of species richness at different resolutions. Global Ecology and Biogeography, 21, 935-944.

Pottier, J., Dubuis, A., Pellissier, L., Maiorano, L., Rossier, L., Randin, C.F. \& Field, R. (2013) The accuracy of plant assemblage prediction from species distribution models varies along environmental gradients. Global Ecology and Biogeography, 22, 52-63.

Pradervand, J.N., Dubuis, A., Reymond, A., Sonnay, V., Gelin, A. \& Guisan, A. (2013) Quels facteurs influencent la richesse en orthoptères des Préalpes vaudoises? Bulletin de la Société Vaudoises des Sciences Naturelles, 93, 155-173.

R Development Core Team (2014) R: a language and environment for statistical computing. R Foundation for Statistical Computing, Vienna. Available at: http://www.R-project.org/

Shipley, B., Vile, D. \& Garnier, E. (2006) From plant traits to plant communities: a statistical mechanistic approach to biodiversity. Science, 314, 812-814.

Soberon, J. \& Nakamura, M. (2009) Niches and distributional areas: concepts, methods, and assumptions. Proceedings of the National Academy of Sciences USA, 106, 19644-19650.

Steinmann, K., Linder, H.P. \& Zimmermann, N.E. (2009) Modelling plant species richness using functional groups. Ecological Modelling, 220, 962-967.

Stone, L. \& Roberts, A. (1990) The checkerboard score and species distributions. Oecologia, 85, 74-79.

Thuiller, W., Georges, D. \& Engler, R. (2014) Biomod2: ensemble platform for species distribution modeling. Version 3.1-48. Available at: http://cran.r-project.org/web/packages/ biomod2/index.html (accessed 6 january 2015).
Turner, J.R.G., Gatehouse, C.M. \& Corey, C.A. (1987) Does solar energy control organic diversity? Butterflies, moths and the British climate. Oikos, 48, 195-205.

\section{SUPPORTING INFORMATION}

Additional supporting information may be found in the online version of this article at the publisher's web-site.

Appendix S1 Variables utilized in the models for single species and in the richness models.

Appendix S2 Thresholds applied to derive binary species distribution model predictions.

Appendix S3 Supplementary methods.

Appendix S4 Supplementary results: evaluation of species distribution models.

Appendix S5 Supplementary results from the co-occurrence analysis: $C$-score.

Appendix S6 Supplementary results from the co-occurrence analysis: checkerboard units index for species pairs.

Appendix S7 Supplementary results for the differential implementation of the single steps of the SESAM framework.

\section{BIOSKETCHES}

Manuela D'Amen is a postdoc and Jean-Nicolas Pradervand is a $\mathrm{PhD}$ student in the spatial ecology group at the University of Lausanne (http://www.unil.ch/ecospat). This group, led by Antoine Guisan, specializes in spatial modelling of species, diversity and community distributions, using empirical data, statistical models and dynamic approaches. There is a strong focus on the use of models and their predictions to support conservation management.

Editor: Adriana Ruggiero 